\title{
Cerebral Localization of Chronic Lymphocytic Leukemia Simulating Progressive Multifocal Leukoencephalopathy: The Lessons from a Case
}

\author{
T. M. Sandouno ${ }^{*}$, S. Chebrek2 ${ }^{2}$ C. Tchonko², G. Pichancourt ${ }^{3}$, L. Giovannetti2, H. Zerazhi' \\ Romain Appay4, H. Lepidi', H. Bachir'1, S. Hamaz', H. B. Alaoui', K. Serraj', B. Slama² \\ ${ }^{1}$ Internal Medicine Department, Mohammed VI University Hospital Center, Mohammed I University, Oujda, Morocco \\ ${ }^{2}$ Onco-hematology department, Henri Duffaut Hospital Center, Avignon, France \\ ${ }^{3}$ Infectiology Departement, Henri Duffaut Hospital Center, Avignon, France \\ ${ }^{4}$ Department of Pathological Anatomy and Neuropathology, Timone University Hospital Center, Marseille, France \\ Email: ^sandouno.marc89@gmail.com
}

How to cite this paper: Sandouno, T.M., Chebrek, S., Tchonko, C., Pichancourt, G., Giovannetti, L., Zerazhi, H., Appay, R., Lepidi, H., Bachir, H., Hamaz, S., Alaoui, H.B., Serraj, K. and Slama, B. (2020) Cerebral Localization of Chronic Lymphocytic Leukemia Simulating Progressive Multifocal Leukoencephalopathy: The Lessons from a Case. Case Reports in Clinical Medicine, 9, 96-106.

https://doi.org/10.4236/crcm.2020.94015

Received: February 2, 2020

Accepted: April 6, 2020

Published: April 9, 2020

Copyright $\odot 2020$ by author(s) and Scientific Research Publishing Inc. This work is licensed under the Creative Commons Attribution International License (CC BY 4.0).

http://creativecommons.org/licenses/by/4.0/

\begin{abstract}
Background: Central neurological involvement is the most frequent extra hematological manifestation of chronic lymphocytic leukemia; it is multifactorial and rarely due to a cerebral localization of the disease. We report a case of cerebral localization of chronic lymphoid leukemia whose clinical and radiological aspects were very suggestive of progressive multifocal leukoencephalopathy. Case Presentation: A 65-year-old patient who was HIV-negative (human immunodeficiency virus), had consulted for bilateral axillary, cervical and inguinal lymphadenopathy associated with major asthenia and hyper lymphocytosis (lymphocyte count was 11 giga/l). Chronic lymphocyticleukemia with TP53 mutation was diagnosed and treatment with Ibrutinib $420 \mathrm{mg} / \mathrm{day}$ was initiated. After 2 months of treatment, the evolution was marked by the onset of neurological disorders whose clinical-radiological presentation and temporal evolution had led to the diagnosis of progressive multifocal leukoencephalopathy. In the absence of virological evidence in the cerebrospinal fluid analysis, a stereotactic biopsy of the brain lesions had been performed, making it possible to formally rule out this infectious hypothesis and to demonstrate cerebral invasion by tumour cells. Immuno-chemotherapy combining RituximabCyclophosphamide-Doxorubicin-Vincristine-Prednisone-Ibrutinib (RCHOPIbrutinib) with intrathecal chemotherapy resulted in a very good clinicalradiological response. Conclusion: The appearance of neurological manifestations in the context of chronic lymphocytic leukemia must systematically lead to a search for a cerebral localization of the disease. In the absence of virological evidence in the cerebrospinal fluid, any suspicion of progressive
\end{abstract}


multifocal leukoencephalopathy in this context should lead to the histological study of brain lesions.

\section{Keywords}

Progressive Multifocal Leukoencephalopathy, Chronic Lymphocytic

Leukemia, John Cunningham Virus, cerebral Localization, Ibrutinib

\section{Background}

Chronic Lymphocytic Leukemia (CLL) is the most common malignant hemopathy after the age of 60 . It is characterized by a monoclonal proliferation of mature B lymphocytes infiltrating the bone marrow and the lymphoid organs, responsible for hyper lymphocytosis in the blood with or without tumor syndrome (lymphadenopathy, hepatosplenomegaly) [1]. Outside of these classical sanctuaries, extra-hematological tumor infiltration is a rare situation.

Although central neurological involvement is one of the most common extrahematological manifestations in CLL, it is rarely an expression of a cerebral localization of the disease, as a variety of etiologies may be responsible for central neurological manifestation in CLL [2].

Progressive multifocal leukoencephalopathy (PML) is a central nervous system infection caused by the John Cunningham Virus (JCV) and promoted by immunosuppression. A classic complication of HIV, PML is also found in lymphoproliferative syndromes where it can be responsible for non-specific neurological manifestations with a radiological expression that is most often quite evocative [3].

Because of the highly variable therapeutic implications depending on the etiology, certainty diagnosis of neurological manifestations in CLL is of highly critical importance.

We report a case of cerebral localization of CLL whose clinical-radiological and evolutionary aspects were very suggestive of progressive multifocal leukoencephalopathy.

\section{Case Presentation}

A 65-year-old diabetic patient, HIV negative, in good general condition (WHO 0), consulted in January 2018 for permanent, bilateral and symmetrical cervical, axillary and inguinal supra-centimetric adenopathies which had appeared 1 month earlier and were evolving in a context generalized asthenia without weight loss.

On clinical examination, the lymphadenopathies were isolated without hepatosplenomegaly. Blood cell count showed isolated hyper lymphocytosis at 11 giga/l. The blood smear identified small mature lymphocytes with Gumprecht shadows and lymphocyte immunophenotyping gave a Matutes score of 4/5 (CD5+/ CD23+/FMC7-/CHL weak/CD79b+). The diagnosis of Chronic Lymphocytic 
Leukemia B (CLL B) at stage B of Binet's prognostic classification was retained. The TP53 mutation was positive on cytogenetic analysis.

Due to debilitating asthenia, treatment for CLL was initiated; in February 2018 Ibrutinib was started at a dose of $420 \mathrm{mg} /$ day. Two months later the patient was hospitalized for seizures with sudden tetraparesis. Spinal magnetic resonance imaging (MRI) was normal, but subcortical white matter abnormalities were seen on brain MRI on T1, Fluid Attenuation Inversion Recovery (FLAIR) and DIFFUSION sequences (Figure 1); the hepatic, renal and metabolic workup was free of abnormalities. The topography and appearance of the brain lesions, particularly the lack of contrast enhancement, associated with the clinical and therapeutic context, suggested the possibility of progressive multifocal leukoencephalopathy (PML) or herpetic meningoencephalitis. The cytological and biochemical analysis of the cerebrospinal fluid (CSF) was negative (3 elements/ $\mathrm{mm}^{3}$ ); the Polymerase Chain Reaction (PCR) of JCV and Herpes Simplex Virus (HSV) was negative in CSF. Based on this PML hypothesis and despite the negativity of the PCR of JCV, Ibrutinib was discontinued and an anti-epileptic treatment was introduced and maintained over the long term to ensure effective control of seizures.

2 months after stopping ibrutinib, the patient was again hospitalized for acute confusional syndrome. A new cerebral MRI revealed an extension of the parenchymatous lesions and the appearance of marked uptake of contrast (Figure 2). Again, both the CSF analysis and the PCR of JCV in the CSF were negative. Hematologically, the lymphocyte count was normal, but lymph node activity was noted. The worsening of the clinical picture, the extension and the contrast enhancement of the cerebral lesions following the discontinuation of ibrutinib, led to the hypothesis of an immune reconstitution inflammatory syndrome (IRIS). Systemic corticosteroid therapy was started immediately at a dose of $1 \mathrm{mg} / \mathrm{kg} /$ day but was discontinued after 4 weeks due to the glycemic imbalance

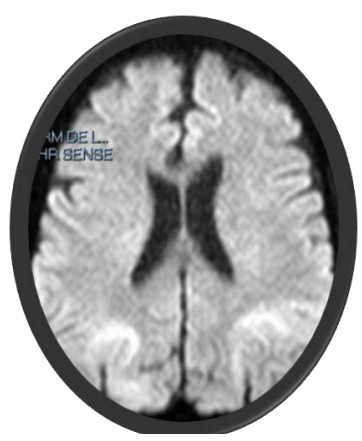

(a)

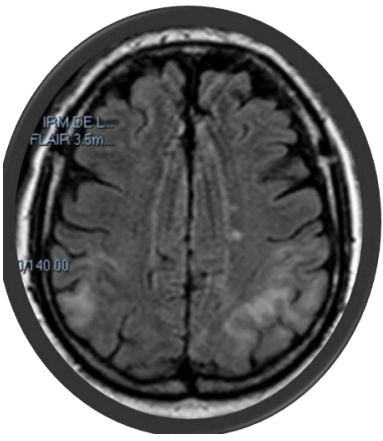

(b)

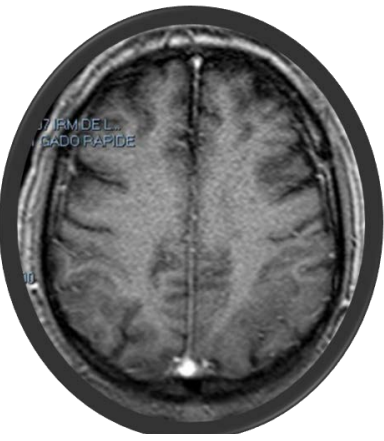

(c)

Figure 1. Appearance of the first neurological symptoms. Brain MRI. Lesions are indicated by the blue arrows. (a) Diffusion sequence: bilateral parieto-occipital hyperintensity, involvement of U-shaped fibers; (b) Axial FLAIR: biparietal hypersignal with U-shaped fibers; (c) T1 axial + gadolinium: hypo-parieto-occipital signal, absence of enhancement of the lesion. 


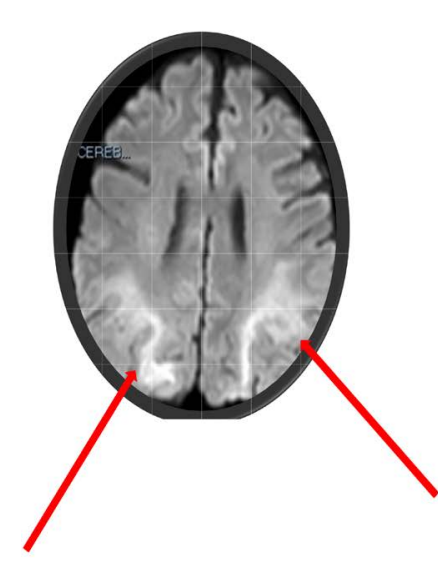

(a)

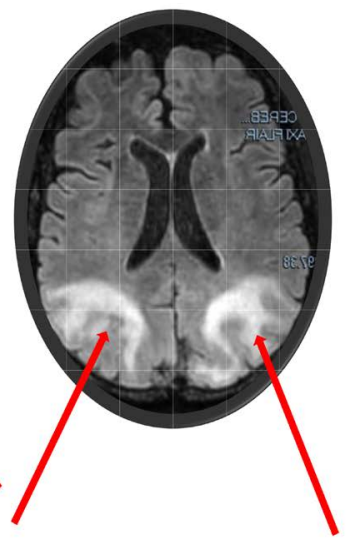

(b)

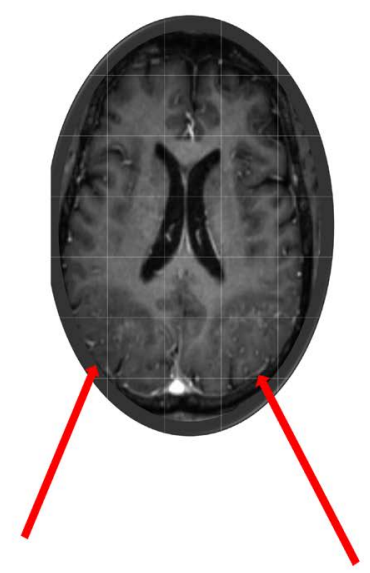

(c)

Figure 2. Worsening of the clinical picture. Cerebral MRI: worsening of neurological lesions. Lesions are indicated by the red arrows. (a) Diffusion Sequence: bilateral parieto-occipital hypersignal, involvement of U-shaped fibers; (b) FLAIR axial: bilateral parieto-occipital hypersignal; (c) T1 axial + gadolinium: bilateral parieto-occipital contrast enhancement, which may indicate an inflammatory response secondary to immune restoration.

and its ineffectiveness on neurological symptoms and tumor mass. 1 month later, faced with the therapeutic impasse and the lack of diagnostic evidence, we decided to perform a stereotaxic biopsy for histological study. This revealed cortical-subcortical tumor infiltration by mature lymphoid elements strongly expressing $\mathrm{CD} 20, \mathrm{CD} 5, \mathrm{CD} 23$ and $\mathrm{BCL} 2$, with perivascular or intra parenchymal topography. There was no histopathological argument for PML (Figure 3).

Systemic immuno-chemotherapy was instituted as follows: 4 intrathecal injections of cytarabine and hydrocortisone, 6 courses of RCHOP-Ibrutinib (Rituximab, Cyclophosphamide, Doxorubicin, Vincristine, and Prednisone) followed by maintenance with Ibrutinib. From the third course of chemotherapy, the clinical response was spectacular, marked by a complete disappearance of the adenopathies and a neurological recovery with a resumption of walking and patient's autonomy. The control MRI at the end of the chemotherapy showed a clear regression of the brain lesions (Figure 4). 3 months after the end of chemotherapy, the patient is still in remission under maintenance treatment with Ibrutinib.

\section{Discussion}

Central neurologic involvement is one of the most common extra-hematologic manifestations encountered in CLL. It is multifactorial; it can be the direct expression of a cerebral localization of tumor lymphocytes or secondary to various infectious, inflammatory, vascular, neoplastic, metabolic or other etiologies [2]. In a study published in 2016, concerning 172 patients followed for CLL who were investigated for central neurological symptoms, approximately $80 \%$ of the cases were due to an etiology other than CLL [2]. Autopsy series revealed that the central nervous system was completely asymptomatically infiltrated in a 


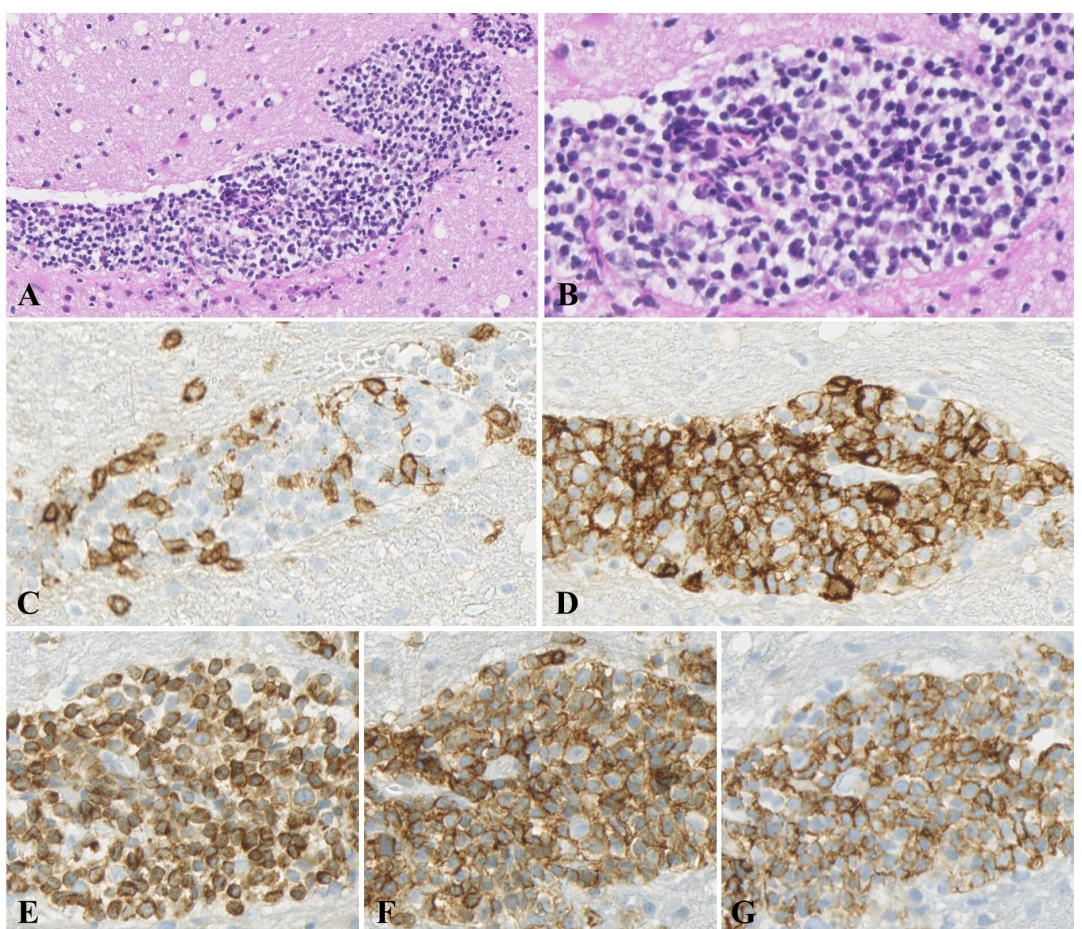

Figure 3. Lymphoid infiltrates of perivascular or intraparenchymal topography (A. HPS $\times 20$ ) represented by small cells, of mature aspect, devoid of mitotic activity and atypical character (B. HPS, $\times 40$ ). CD3 targets an associated reactive T lymphocyte component (C). Lymphomatous cells strongly express CD20 (D), protein Bcl2 (E), CD5 (F) and CD23 (G).

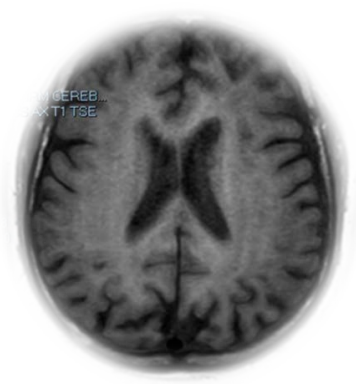

(a)

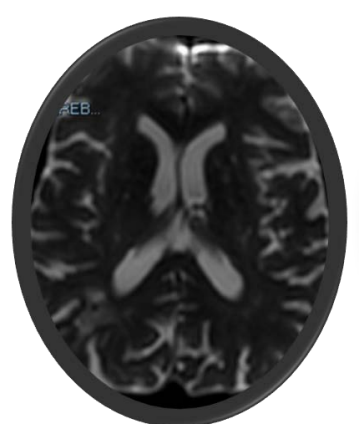

(c)

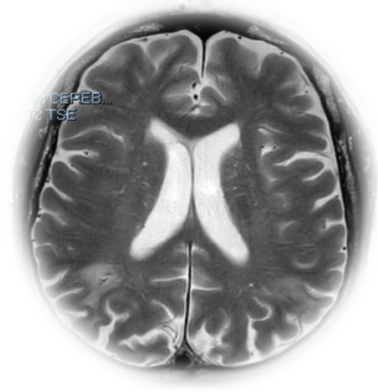

(b)

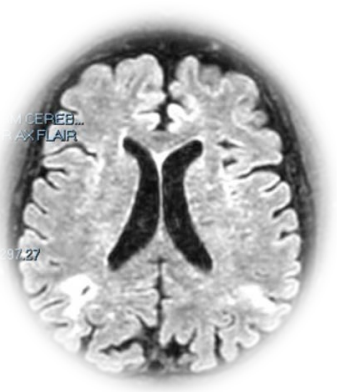

(d)

Figure 4. Brain MRI after 6 courses of chemotherapy. Significant regression of brain damage. (a) Axial T1 sequence; (b) Axial T2 sequence; (c) Diffusion sequence; (d) Axial flair. 
proportion varying from $7 \%$ to $71 \%$ [4] [5]. This relatively high frequency of asymptomatic localizations on the one hand, and the considerable proportion of non-CLL etiologies on the other, justify the difficulty of the etiological approach in this context.

The clinical-radiological picture presented by our patient mainly evoked the hypothesis of PML. This hypothesis has been supported by the immunosuppression inherent in CLL and the use of a treatment that promotes apoptosis of lymphocytic cells.

PML is a central nervous system infection caused by the JCV through immunosuppression. It is frequently seen in HIV-positive patients, but can occur in patients with lymphoproliferative diseases or those treated with immunosuppressive drugs or certain targeted therapies such as Rituximab [3] [6]. In case of iatrogenic origin, it usually occurs after prolonged exposure, but rare cases of early onset are described in the literature [7] [8]. Ibrutinib, despite insufficient evidence, has been associated with cases of PML in some series [7] [9].

Cognitive and visual impairments, gait and coordination disorders, and dysarthria are the main clinical manifestations of PML, but seizures may be present in some cases [6] [10]. MRI is an essential tool in the diagnostic process, showing lesions of the subcortical white matter, in hyper signal T2 and FLAIR and in hypo signal T1. Classically, they are not enhanced after gadolinium injection and mainly concern arched fibers or U-shaped fibers with extensive and multifocal topography, which are particularly localized to posterior and temporo-parietal regions [6] [8]. The diagnosis of certainty is based on the detection of the JCV in the CSF or brain tissue. It should be noted, however, that CSF negativity does not rule out the diagnosis of PML and a histological study is essential to formally conclude [11].

Its management is not codified; no treatment has been proven for this indication, as restoration of immunity remains the only condition capable of controlling viral replication [6].

In addition to the initial clinical and radiological presentation, the occurrence of an immune reconstitution inflammatory syndrome (IRIS), which was suggested by the worsening of the clinical picture and the change in radiological aspect (extension of the lesions with contrast enhancement) after discontinuation of ibrutinib, further strengthened the diagnosis of PML initially suspected.

IRIS is an exuberant and deregulated inflammatory response to an infectious or non-infectious antigen. It results in the abrupt aggravation of a pre-existing condition or the sudden appearance of atypical clinical manifestations in the context of immune restoration. Classically found in cases of HIV infection, IRIS can occur in other pathological circumstances. It can be induced by reduction or abrupt discontinuation of immunosuppressive therapy. Corticosteroid therapy is the most commonly used treatment in this context [6] [12].

The diagnosis of PML was based on highly compatible clinical and radiological data, but we lacked virological or histological evidence of JCV imputability. 
The lack of diagnostic evidence on the one hand and the rapid progression of adenopathies on the other made each of the hypotheses plausible: tumor infiltration by CLL, PML or other. It is in this context that the stereotaxic biopsy of an occipital lesion was performed; Histology revealed tumor infiltration of the white matter and the cortex by cells of known CLL with high expression of CD20, CD5, CD23 and Bcl2. However, it must be stressed that this discovery does not resolve one question: is it an extension of the disease complicating its evolution or rather an initially asymptomatic localization revealed by the therapeutic effect of Ibrutinib, when we know the capacity of this drug to cross the blood-brain barrier [13]? On the other hand, this discovery, in conjunction with our patient's CSF analysis, highlights the low sensitivity of CSF in the diagnosis of a cerebral localization of CLL. A previously cited study had shown the discrepancy between CSF cytology and cerebral histology: among 131 patients with negative cytology, $11 \%$ had a positive histology; finally among 31 patients with positive cytology, $58 \%$ had an alternative diagnosis to histology [2]. In the latter case, the presence of tumor lymphocytes in the CSF could be secondary to contamination with peripheral blood at the time of collection. As a result, CSF analysis is of low positive and negative predictive value for the diagnosis of a cerebral localization of the CLL.

Cases of CNS infiltration in CLL are described in the literature. The analysis of these cases allows us to observe a mean age of 64.5 years with a slight predominance of the male sex, a very heterogeneous clinical symptomatology with a predominance of cranial neuropathy followed by non-specific manifestations such as headache, cognitive disorders, coordination disorders or convulsions. The time from diagnosis of CLL to the onset of neurologic complications varied widely from patient to patient, ranging from a few weeks to several years; however, neurologic complications are rarely a mode of revelation of CLL [14] [15] [16]. The non-specific nature of the symptoms justifies the diagnostic delay with an average delay of 5 months. The leptomeningeal localization was the most represented, confirmed most often by the cytological study and CSF flow cytometry [14] [15]. No correlation has been established between the stage of CLL and the occurrence of neurological complications; indeed neurological localization has been diagnosed in patients in the early stage of the disease or in completely asymptomatic patients with no systemic CLL activity [17]. To date, no study has been able to identify a risk factor for neurological progression of CLL, although soluble CD27 and the study of CD49d/CD82 expression have been proposed as biomarkers of risk [16] [18]. Overall, a favourable therapeutic response was obtained [14] [15].

In addition to the common characteristics it shares with the cases described in the literature, our case has the following particularities:

- The occurrence of neurological complications during the treatment phase

- A relatively infrequent mode of disclosure compared to previously published cases. 
- The hematological response under targeted therapy (normal blood count, disappearance of lymphadenopathy) coupled with the absence of tumor cells in the CSF made a neurological localization of the disease very unlikely.

- Ibrutinib, the targeted therapy used, has pharmacological properties that allow it to cross the blood-brain barrier and therefore exert its anti-tumor effect [13]; this, in view of the hematological response obtained, would make a neurological localization of CLL highly unlikely.

- The therapeutic implications are radically opposed in this context: while the diagnosis of CLL with neurological invasion requires, local or systemic chemotherapy, PML contraindicates all chemotherapy and requires immune restoration as the only therapeutic weapon recognized to date. In our case, the PML hypothesis would have been very detrimental for our patient who, let us recall, had CLL with a $17 \mathrm{p}$ deletion.

Furthermore, this case raises some questions: how can we explain the neurological impairment in CLL-patients in hematological response to ibrutinib, which is also an effective molecule in the CNS? How to explain the clinical picture and the morphological aspects observed on MRI?

Given the high frequency of asymptomatic CLL cerebral localizations, we believe that in our case the central nervous system was probably already silently infiltrated before the start of ibrutinib treatment. The efficacy of ibrutinib on peripheral tumor mass makes the opposite hypothesis unlikely. This invasive capacity of CLL cells is thought to be due to the dysregulation of certain integrins involved in cell migration [16].

At first glance, the morphological aspects observed on MRI would seem to correspond to a local inflammatory phenomenon as shown by MRI contrast enhancement reflecting the rupture of the blood-meningeal barrier. Should this be seen as a consequence of the action of ibrutinib? This seems implausible to us, since from a physiological point of view, the apoptosis resulting from the anti-tumour effect of ibrutinib does not induce an inflammatory reaction.

The treatment of CLL brain localizations is not codified; the few available data are empirical and relate to clinical cases or series of patients. The therapeutic strategies used vary from case to case and may or may not combine systemic chemotherapy, intrathecal chemotherapy or radiotherapy [19]. Their efficacy is variable, however there is no data to date to confirm the superiority of one strategy over another; the efficacy of Ibrutinib in this indication is found in some publications [19] [20].

In our case, we had opted for the combination of intrathecal chemotherapy with systemic immunochemotherapy, as follows: 4 intrathecal injections of cytarabine and hydrocortisone, 6 courses of RCHOP + Ibrutinib followed by maintenance with Ibrutinib. A clinical response was obtained after only 3 courses of chemotherapy: complete disappearance of superficial adenopathies, resumption of walking and patient's autonomy. The control MRI performed at the end of the chemotherapy showed a clear regression of the hyper signals. 3 
months after the end of chemotherapy, the patient is doing well and is being maintained on Ibrutinib.

\section{Conclusions}

We infer from this case that the appearance of neurological symptoms or signs in a context of CLL should systematically evoke and search for a neurological localization of CLL regardless of the stage of the disease, its duration of progression, lymphocytes count, clinical presentation, radiological appearance or treatment received. Clinical examination, CSF studies, brain imaging and histology are key means of making the diagnosis.

In the absence of virological evidence in the CSF, any clinical and radiological suspicion of PML in the context of chronic lymphocytic leukemia should lead to the histological study of brain lesions in order to confirm the diagnosis of PML on the one hand and to rule out the possibility of a tumor localization on the other. This distinction is of critical importance because, unlike PML for which there is no effective therapy to date, there are treatments that have been shown to be effective in the management of CLL cerebral localizations.

\section{Acknowledgments}

The authors would like to thank the radiology department of the Henry Duffaut Hospital Center in Avignon for their contribution to the iconography of this article.

\section{Consent}

The patient's oral consent was obtained for the publication of this case and the associated iconography.

\section{Conflict of Interest}

The authors declare no conflicts of interest regarding the publication of this paper.

\section{References}

[1] Kipps, T.J., Stevenson, F.K., Wu, C.J., Croce, C.M., Packham, G., Wierda, W.G., O’Brien, S., Gribben, J. and Rai, K. (2017) Chronic Lymphocytic Leukaemia. Nature Reviews Disease Primers, 3, Article No. 16096. https://doi.org/10.1038/nrdp.2016.96

[2] Strati, P., Uhm, J.H., Kaufmann, T.J., Nabhan, C., Parikh, S.A., Hanson, C.A., Chaffee, K.G., Call, T.G. and Shanafelt, T.D. (2016) Prevalence and Characteristics of Central Nervous System Involvement by Chronic Lymphocytic Leukemia. Haematology, 101, 458-465. https://doi.org/10.3324/haematol.2015.136556

[3] Neil, E.C. and DeAngelis, L.M. (2017) Progressive Multifocal Leukoencephalopathy and Hematologic Malignancies: A Single Cancer Center Retrospective Review. Blood Advances, 1, 2041-2045. https://doi.org/10.1182/bloodadvances.2017008201

[4] Bojsen-Moller, M. and Nielsen, J.L. (1983) CNS Involvement in Leukaemia. An Autopsy Study of 100 Consecutive Patients. Acta Pathologica, Microbiologica, et 
Immunologica Scandinavica. Section A, 91, 209-216. https://doi.org/10.1111/j.1699-0463.1983.tb02748.x

[5] Cramer, S.C., Glaspy, J.A., Efird, J.T. and Louis, D.N. (1996) Chronic Lymphocytic Leukemia and the Central Nervous System: A Clinical and Pathological Study. Neurology, 46, 19-25. https://doi.org/10.1212/WNL.46.1.19

[6] Tan, C.S. and Koralnik, I.J. (2010) Beyond Progressive Multifocal Leukoencephalopathy: Expanded Pathogenesis of JC Virus Infection in the Central Nervous System. The Lancet Neurology, 9, 425-437. https://doi.org/10.1016/S1474-4422(10)70040-5

[7] Bennett, C.L., Berger, J.R., Sartor, O., Carson, K.R., Hrushesky, W.J., Georgantopoulos, P., Raisch, D.W., Norris, L.B. and Armitage, J.O. (2018) Progressive Multi-Focal Leucoencephalopathy among Ibrutinib-Treated Persons with Chronic Lymphocytic Leukemia. British Journal of Haematology, 180, 301-304.

https://doi.org/10.1111/bjh.14322

[8] Tevazzi, E., Ferrante, P. and Khalili, K. (2011) Progressive Multifocal Leukoencephalopathy: An Unexpected Complication of Modern Therapeutic Monoclonal Antibody Therapies. Clinical Microbiology and Infection, 17, 1776-1780.

https://doi.org/10.1111/j.1469-0691.2011.03653.X

[9] Lutz, M., Schulze, A.B., Rebber, E., Wiebe, S., Zoubi, T., Grauer, O.M., Keßler, T., Kerkhoff, A., Lenz, G. and Berdel, W.E. (2017) Progressive Multifocal Leukoencephalopathy after Ibrutinib Therapy for Chronic Lymphocytic Leukemia. Cancer Research and Treatment, 49, 548-552. https://doi.org/10.4143/crt.2016.110

[10] Lima, M.A., Drislane, F.W. and Koralnik, I.J. (2006) Seizures and Their Outcome in Progressive Multifocal Leukoencephalopathy. Neurology, 66, 262-264.

https://doi.org/10.1212/01.wnl.0000194227.16696.11

[11] Berger, J.R., Aksamit, A.J., Clifford, D.B., Larry, D., Koralnik, I.J., Sejvard, J.J., Bartt, R., Major, E.O. and Nath, A. (2013) PML Diagnostic Criteria: Consensus Statement from the AAN Neuroinfectious Disease Section. Neurology, 80, 1430-1438. https://doi.org/10.1212/WNL.0b013e31828c2fa1

[12] Sueki, H., Mizukawa, Y. and Aoyama, Y. (2018) Immune Reconstitution Inflammatory Syndrome in Non-HIV Immunosuppressed Patients. The Journal of Dermatolo$g y, 45,3-9$. https://doi.org/10.1111/1346-8138.14074

[13] Goldwirt, L., Beccaria, K., Ple, A., Sauvageon, H. and Mourah, S. (2018) Ibrutinib Brain Distribution: A Preclinical Study. Cancer Chemotherapy and Pharmacology, 81, 783-789. https://doi.org/10.1007/s00280-018-3546-3

[14] De Souza, S.L., Santiago, F., Ribeiro-Carvalho, M.d.M., Arnobio, A., Soares, A.R. and Ornellas, M.H. (2014) Leptomeningeal Involvement in B-Cell Chronic Lymphocytic Leukemia: A Case Report and Review of the Literature. BMC Research Notes, 7, Article No. 645. https://doi.org/10.1186/1756-0500-7-645

[15] Timmers, N.K.L.M., de Maar, J.S., van Kruijsdijk, R.C.M. and Klein, S.K. (2018) Central Nervous System Localisation of Chronic Lymphocytic Leukaemia, Description of Two Very Distinct Cases and a Review of the Literature. Annals of Hematology, 97, 1627-1632. https://doi.org/10.1007/s00277-018-3329-2

[16] Rojas-Hernandez, C.M., Nemunaitis, J., Marjon, K.D., Bustamante, D., Zhang, Q.Y. and Gillette, J.M. (2017) Chronic Lymphocytic Leukemia with Clinical Debut as Neurological Involvement: A Rare Phenomenon and the Need for Better Predictive Markers. BMC Hematology, 17, Article No. 3. https://doi.org/10.1186/s12878-017-0073-0

[17] Miller, K., Budke, H. and Orazi, A. (1997) Leukemic Meningitidis Complicating Early Stage Chronic Lymphocytic Leukemia. Archives of Pathology \& Laboratory 
Medicine, 121, 524-527.

[18] Van den Bent, M.J., Lamers, C.H., van't Veer, M.B., Sillevis Smitt, P.A., Bolhuis, R.L. and Gratama, J.W. (2002) Increased Levels of Soluble CD27 in the Cerebrospinal Fluid Are Not Diagnostic for Leptomeningeal Involvement by Lymphoid Malignancies. Annals of Hematology, 81, 187-191.

https://doi.org/10.1007/s00277-002-0448-5

[19] Wanquet, A., Birsen, R., Bonnet, C., Boubaya, M., Choquet, S., Dupuis, J., et al. (2017) Management of Central Nervous System Involvement in Chronic Lymphocytic Leukemia: A Retrospective Cohort of 30 Patients. British Journal of Haematology, 176, 37-49. https://doi.org/10.1111/bjh.14387

[20] Wanquet, A., Birsen, R., Lemal, R., Hunault, M., Leblond, V. and Aurran-Schleinitz, T. (2016) Ibrutinib Responsive Central Nervous System Involvement in Chronic Lymphocytic Leukemia. Blood, 127, 2356-2358.

https://doi.org/10.1182/blood-2016-02-697193 